\title{
Driving Trends in Information and Communication Technology - Markets and Technologies
}

\author{
Reda Reda ${ }^{1)}$ and Harmen R. van As ${ }^{2)}$ \\ 1) Siemens AG Austria, Vienna, Austria; ${ }^{2)}$ Vienna University of Technology, Vienna, Austria
}

\begin{abstract}
This paper presents an analytical study of the development of the information and communication technology (ICT) market from the marketing as well as from a technological point of view. The trends and their impact on the information communication networks and consequently smart networks are discussed. Despite the current global economic disaster in almost every single industry, there are still a lot of business opportunities within the ICT industry, e.g., revenue-generating new features, applications and value-added services, soft-switches, plug-and-play network elements, network security, e- and mbusiness, micro-payment systems, personalized blue-tooth bandwidth applications and web-based add-ons. Moreover, there are still enormous business chances to adapt, to optimize, and to exploit in the already existing network-infrastructures in Eastern Europe as well as in the Third World.
\end{abstract}

\section{HARDWARE AND SOFTWARE EVOLUTION}

To understand the ICT evolution and the corresponding market development and trends, we present a short survey of the hardware and software evolution during the last decade and a corresponding prognosis for the next decade. The physicist Niels Bohr once said: "Prediction is very difficult, especially about the future". The following examples display this fact very impressively. Tom Watson Sr., Chairman of IBM, said in 1943, "I think there is a world market for maybe five computers" and Irwin Dorros, Vice President of Long Lines ATT, stated in 1981, "ISDN will be ubiquitous by 1985 ". Despite the mentioned fact that predictions about the future are

The original version of this chapter was revised: The copyright line was incorrect. This has been corrected. The Erratum to this chapter is available at DOI: 10.1007/978-0-387-35584-9_19 
indeed difficult, this section invites to have a look at possible future aspects concerning hardware and software.

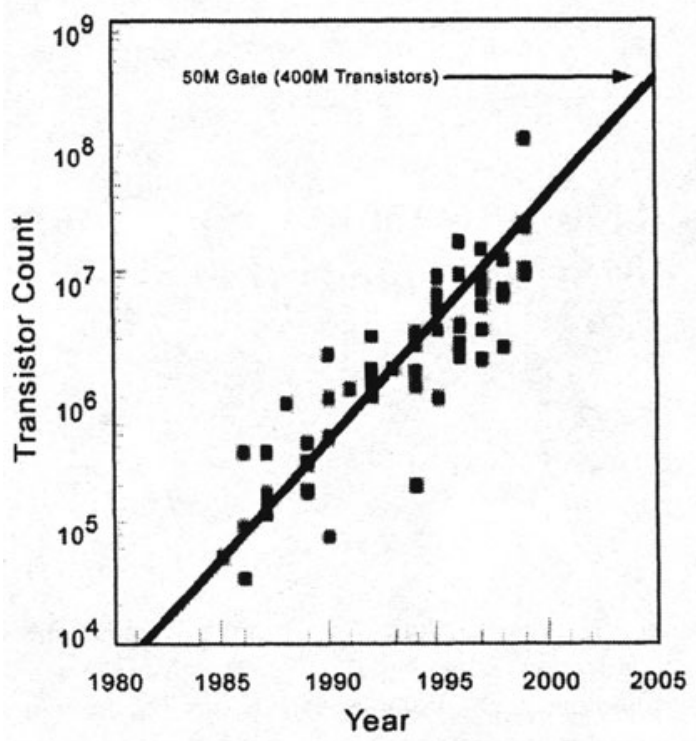

Figure 1. Trends in integrated circuit complexity

\subsection{Hardware Evolution}

Integration density and performance of integrated circuits have gone through an astounding revolution in the last couple of decades. In the 1960s it was predicted that the number of transistors that can be integrated on a single die would grow exponentially with time. This prediction, later called Moore's law, has proven to be amazingly visionary. Its validity is best illustrated by a set of graphs. Figure 1 plots the integration density of integrated circuits and Figure 2 displays the integration density of memory as a function of time. As can be observed, integration complexity doubles approximately every one to two years. As a result, memory density has increased by more than a thousand-fold since 1970. An intriguing case study is offered by the microprocessor. From its inception in the early seventies, the microprocessor has grown in performance and complexity at a steady and predictable pace (Figure 3 for Moore's law). Clock frequencies double every three years and have already reached into the $\mathrm{GHz}$ range. This is illustrated in Figure 4, which plots the microprocessor trends in terms of complexity and performance at the beginning of the 21 st century. An 
important observation is that, as of now, these trends have not shown any signs of a slow-down.

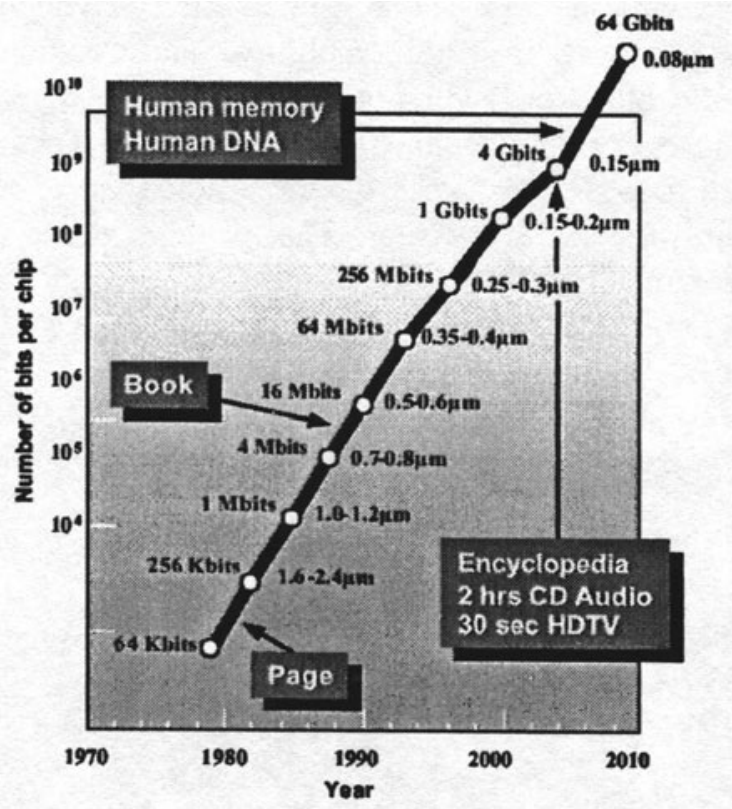

Figure 2. Trends in memory complexity

Beginning with Moore's law to the vanishing of today's personal computers, the following part tries to deliver an overview of various technological trends in the field of future hardware. The microprocessor performance will still double every 18 months, or even faster - more likely every 14 months. This is also known as Moore's Law. In addition, transmission capacity will even double every 12 months. Considering the Internet, its number of addresses will double every six months. To solve the problem of this ever-increasing demand of IP addresses IPv6 was introduced, through which it would be possible to address not only every single chip in a interplanetary Internet, but even every single atom in the universe. In this respect, Metcalfe's Law says that a network's usefulness, or value, goes up as the square of the number of its users, i.e., with only one user a network is no utility; thousand users form a corporate Local Area Network (LAN); America Online (AOL) has 29 million customers; and everyone accessing the Internet means 6 billion users. Most importantly, exponential growth means exponential benefit. Miniaturization and performance will be more and more the main driving factors for the hardware market. Other facts are that portable mobile devices will gain multimedia capabilities, that worldwide networking and supercomputing will 
come along with a considerable growth in bandwidth, and that smart card applications such as multi-functional and programmable devices will evolve on the market. We will see an ascending integration of telephone, TV and personal computer together with mobility and complex network. Convergence as the buzzword will not only comprehend technologies or networks, but also every single industry and business. Today, 30 million chips are produced for PCs every year, whereas more than 250 million chips are built into other devices every year. The average American household comprises approximately 40 microprocessors giving an 'ambient intelligence', and familiar surroundings become an interface to a worldwide communication infrastructure. Today's PCs start to vanish.

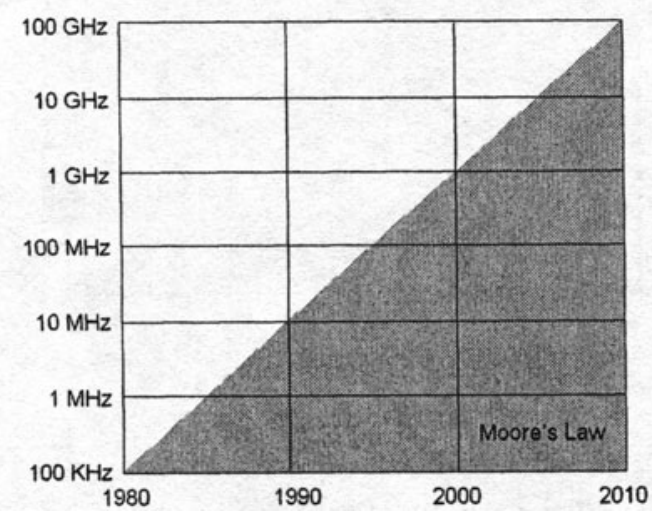

Figure 3. Microprocessor clock frequency: 100x per decade

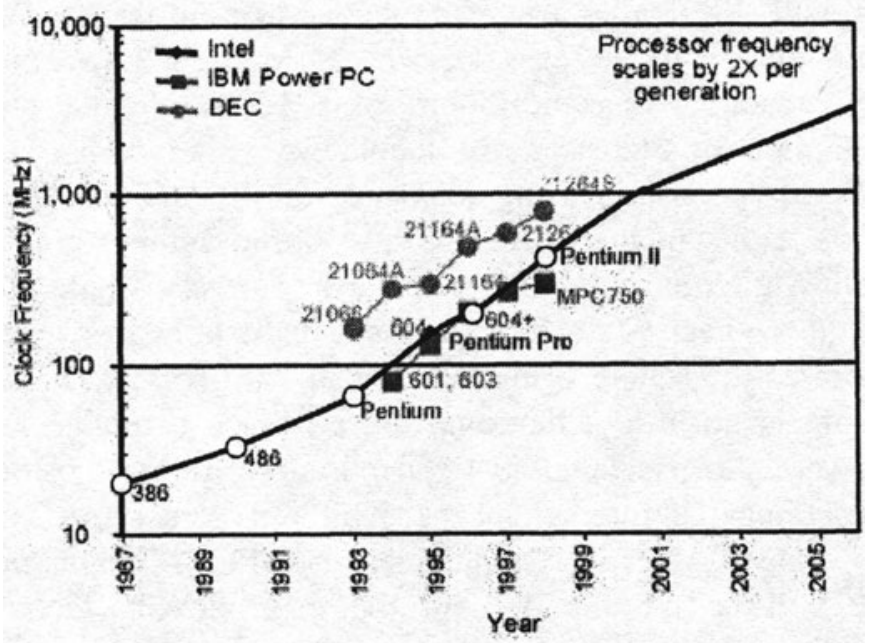

Figure 4. Trends in clock frequency 
The examples of hard disk capacity and CPU speed illustrate these enormous achievements. The hard disk capacity increases by a factor 100 per decade, whereas access speed only increases by a factor 10 in the same period. This implies that the time to scan an entire hard disk increases by a factor 10 every decade. Figures 5 and 6 show the incredible development of microprocessors by means of different layout-designs from two Intel microprocessor generations.

$\begin{array}{rrcrr}1989 & 20 \mathrm{MB} & \$ 650 & 1974 & 2 \mathrm{MHz} \\ 1994 & 345 \mathrm{MB} & \ldots & 1985 & 16 \mathrm{MHz} \\ 1999 & 50 \mathrm{~GB} & \ldots & 1994 & 75 \mathrm{MHz} \\ 2000 & 60 \mathrm{~GB} & \ldots & 1999 & 700 \mathrm{MHz} \\ 2001 & 100 \mathrm{~GB} & \$ 250 & 2001 & 2000 \mathrm{MHz}\end{array}$

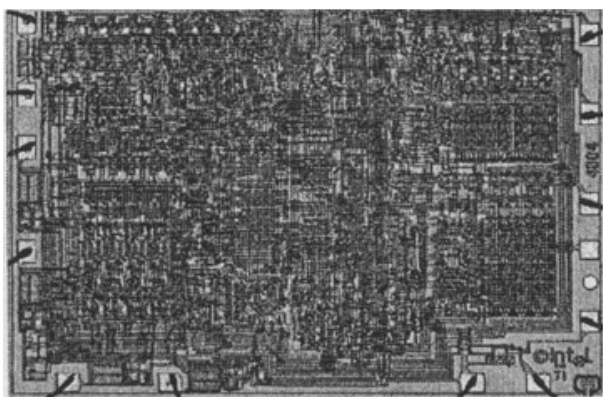

Figure 5. Intel 4004 microprocessor $(1971,108 \mathrm{kHz}, 4 \mathrm{kB}$ of address. memory)

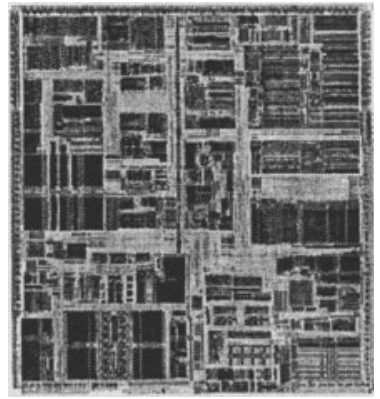

Figure 6. Intel Pentium II microprocessor $(1997,233 \mathrm{MHz}, 4 \mathrm{~GB}$ of address. memory)

\subsection{Software Trends}

The following part gives a survey of some evolution milestones in the field of software.

Media: Digitization (and increase of processing power and memory size needed) of all different types of media leads to an enormous increase of information available and many different forms of direct electronic communication. Consumers will also digitize their own media such as photographs and video.

System architecture: Worldwide distributed systems allow to maintain information in a decentralized manner, enabled by layered and modular architectures, where hardware and communication specific details are 
hidden. This reflects the demand for more efficient heterogeneous network management systems.

Standard Software: Operating systems, programming languages with graphical programming tools and databases tend to follow more and more a homogenous paradigm. In operating systems, we will see the survival of just two systems, UNIX and Windows. This also leads to an increased reusability of software modules and a better interoperability of systems.

Software process: The whole software industry benefits from the homogenous paradigm. New approaches such as participatory designs, workflow procedures, computer supported cooperative work, and reusable components are common now. But good software design takes time. The tools needed only improve in small steps because object-oriented software design is not achieving its promise. Software development costs increase. For these reasons, software projects take more time and cost more money, since documentation and maintenance is expensive, therefore quickly leading to legacy systems.

Applications: User-assisting applications are needed in an increasingly number of fields. But they have a higher time-to-market and competitive pressure leads to software, which is full of errors, decreasing quality and reliability. Modeling of human behavior and thinking is still a hard problem, creating the biggest gap between expectation and reality.

User interfaces: Great developments from character-based terminal to window-based interfaces (WYSIWYG - What You See Is What You Get) have been achieved, now followed by multi-modal interfaces, virtual-reality, and speech recognition. But interfaces are still a hot topic. It needs interdisciplinary approach, including psychology and sociology. Software must become fault-tolerant, self-repairing, remotely manageable, and remotely deployable.

Finally, Nathan's law says software is a gas. It expands to fill the volume available. Concluding, Figure 7 shows the well-known hardware/software life-cycle, which is the main driving factor for future software and hardware development. 


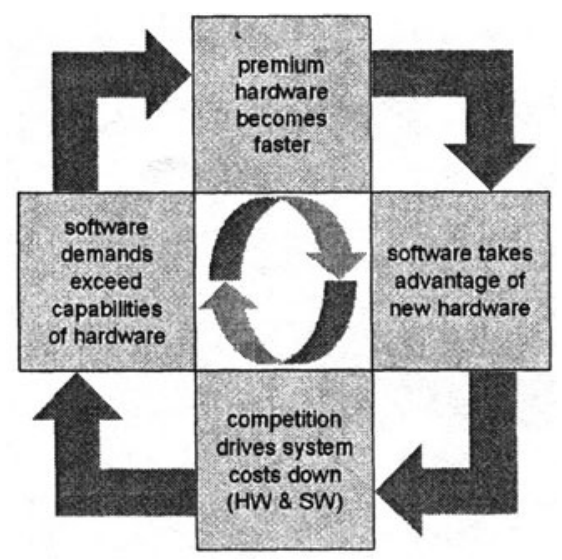

Figure 7. Hardware / software life-cycle

\subsection{Hardware and Software Research Trends}

Research in the field of hardware and software technology will supposedly focus on the following topics in the near future: interfaces, e.g., multilingual e-commerce applications, personalization, and natural interactivity; embedded systems; integrating and linking fixed and mobile communication; distributed computing; architectures for information integration; as well as privacy and security

\subsection{Taking a Decade-Leap in Time}

The following part takes into consideration the facts and predictions stated above and tries to predict what hardware and software of the next decade may look like. A personal computer of the next decade might have an 1,5 THz processor, 100 GByte of memory, a 10 TByte hard disk and a wallsized display with $8192 \times 8192$ pixels. A Server of the next decade might consist of an interconnected system of 64 processors at $1,5 \mathrm{THz}, 5 \mathrm{TByte}$ of memory, a 1 PByte hard disk, and a 100-Gbit/s Ethernet network interface. Operating systems in the next decade might show that Microsoft could no longer afford to enhance Windows, because the complexity became overwhelming; companies like IBM, HP, and Sun have abandoned their own Unix for Linux with private extensions, since the cost of building an own operating system became too high; and devices have small, specialized operating systems. 
$\sum$ Information- und Communication Technology (ICT) is the main driving force in the economy

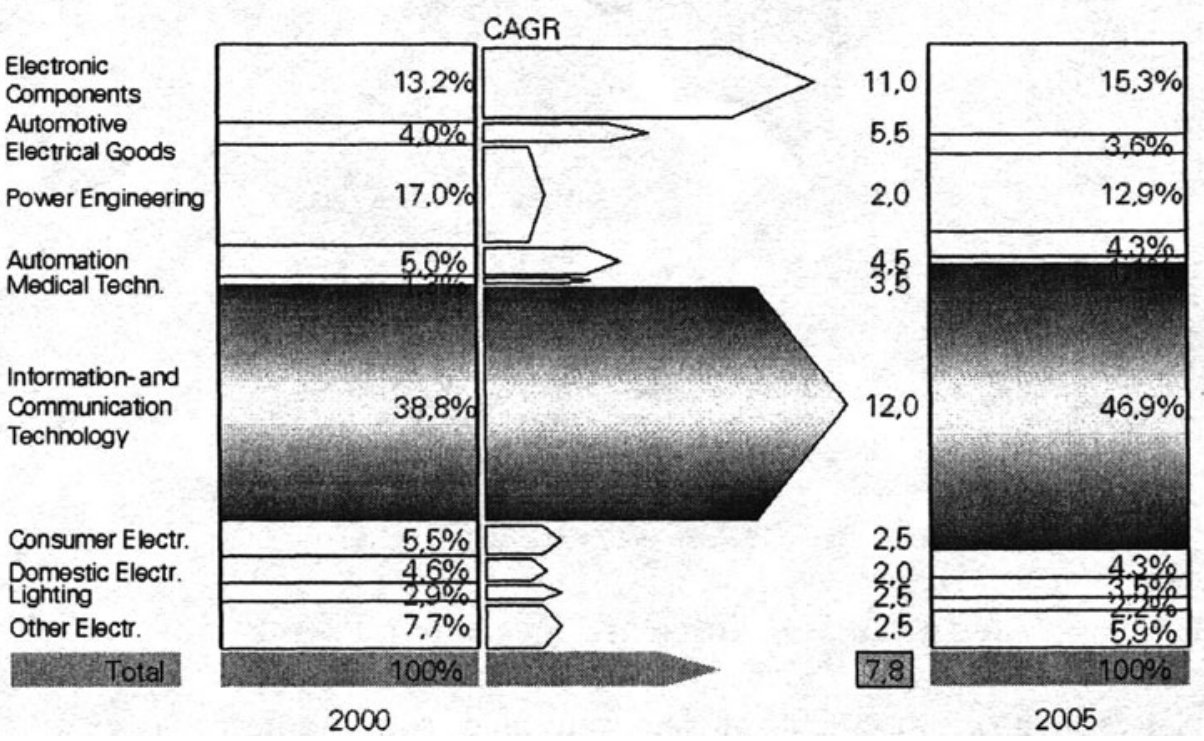

Figure 8. World electronics market - development 2000-2005

\section{ICT MARKET POTENTIAL}

The development of the information and communication technology (ICT) market over the recent years has been exceptional, perhaps even unique in the history of industrial change. According to recent studies, the ICT world market in 2001 is expected to exceed the 1 Billion Euro mark (approx. 1,106 Billion Euro), with a quite impressing combined annual growth rate (CAGR) of $12 \%$. The ICT market represents almost $39 \%$ of the total world electrical market. Thus, this is the main driving force in the progress of the electrical industries. Figure 8 illustrates the breakdown of the total market of electrical equipment and gives a prognosis for the year 2005.

\subsection{Worldwide ICT Market Trends}

Considering the relative growth of the ICT market, a slow-down and uncertainty has been observed in the second half of 2001, but the European market growth is still higher than in the US and Japan, as can be derived from Figure 9. Furthermore there are still good ICT business chances in Eastern Europe and the Third World, despite the global economical crises 


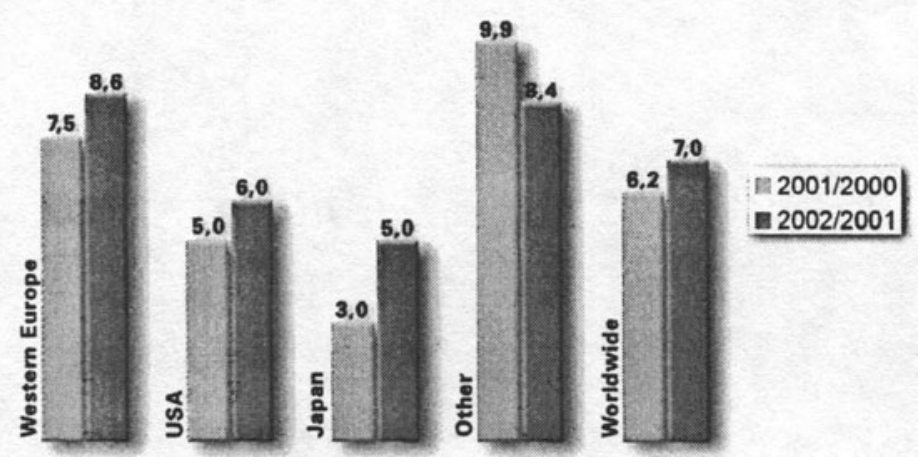

Figure 9. Worldwide IT market growth by region 2001-2002 (\%)

The major factors effecting this slowdown are the global economic slowdown and the dotcom disappearance; the heavy 3G (Third Generation) license fees cutting or delaying carriers investment programs; delays in availability of GPRS and 3G, coupled with relative saturation of the GSM market; pricing and tariff competition squeezing revenues streams; low market acceptance of ASPs (Application Service Providers) and emarketplace models; ongoing skill shortage hampering e-business development.

From the other side, the major factors effecting the ICT market growth are: from dotcom euphoria to real e-business for "brick-and-mortar" companies; increasing adoption of Internet-centric solutions for e-business; integrated services moving from back-office into front-office operations; unmetered Internet access favoring consumer and professional usage; growing mobile data expectations; broadband (ADSL and WLL) diffusion driving Internet and multimedia applications and services; e-government programs notably at local community level.

\subsection{ICT Market Growth in Western Europe}

The ICT market for Western Europe showed a serious slow-down in growth in 2001. This trend will continue for the telecommunications market in 2002, whereas the IT market will be able to slightly increase its growth. More interesting seems the fact, that in 2001 and 2002 the IT market overtakes the telecommunications market with respect to growth potential for the first time ever, as can be derived from Figure 10. 


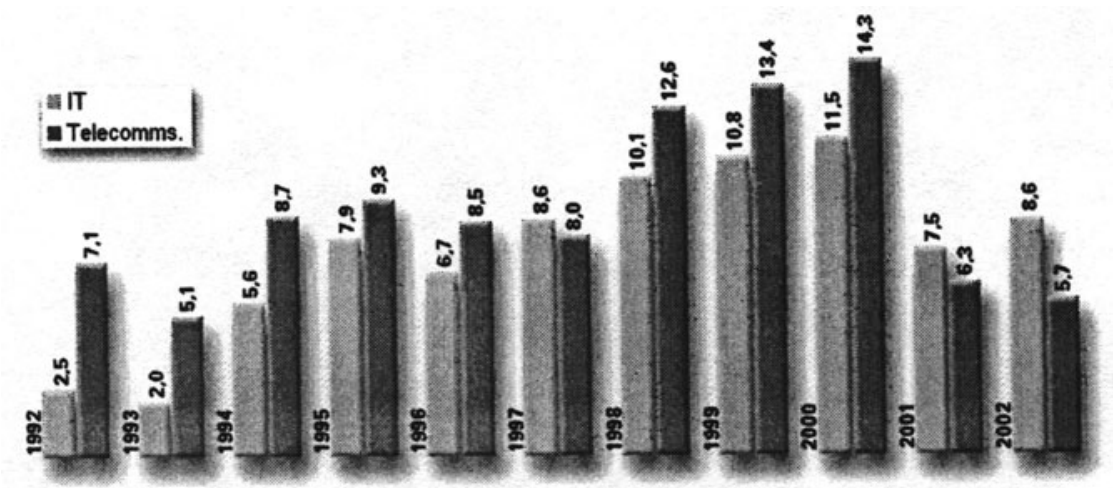

Figure 10. Western Europe ICT market: actual growth and forecast 1992-2002 (\%)

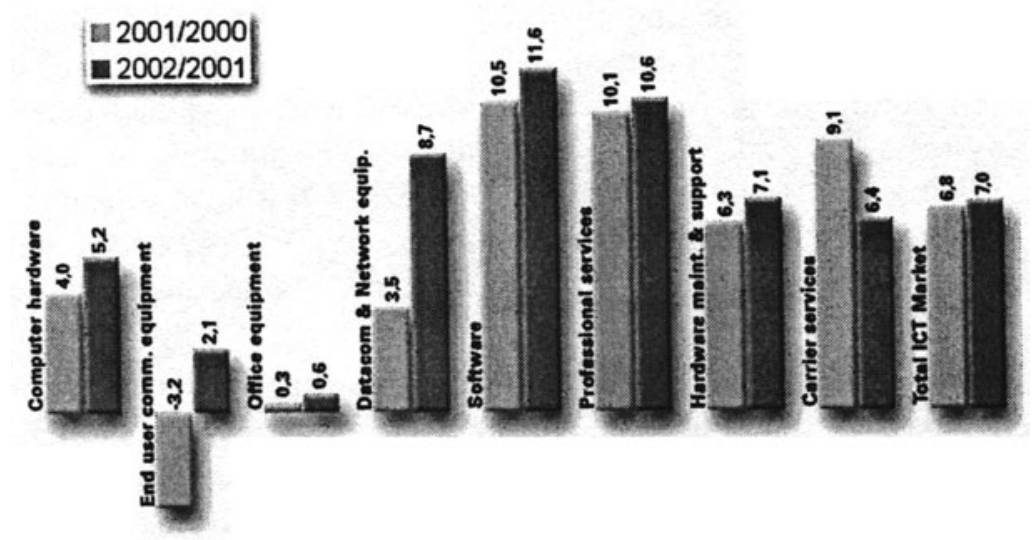

Figure 11. Western Europe ICT market growth by product segment 2001-2002 (\%)

Dividing the Western European ICT market into its product segments shows some interesting facts (Figure 11): All product segments including computer hardware, network equipment, end user communications equipment (which really had a bad year in 2001) and software will be able to increase their market growth in the year 2002. But carrier services will decrease its growth by approximately $30 \%$ in 2002 .

Figure 12 shows a daring look into the future of access platforms in European homes up to the year 2010. As expected the plain old telephone service (POTS) will loose its importance with regard to home Internet access and decrease to approximately $12 \%$ in the year 2010 . ISDN access should vanish by the year 2005 , mainly because of the tremendously increasing market share of DSL, Coax-Cables, Fibre-Hybrid and Fibre-Pure accesses. Finally, other technologies like satellite access or FWA will not gain importance at all and stay below $2 \%$ in 2010 . 


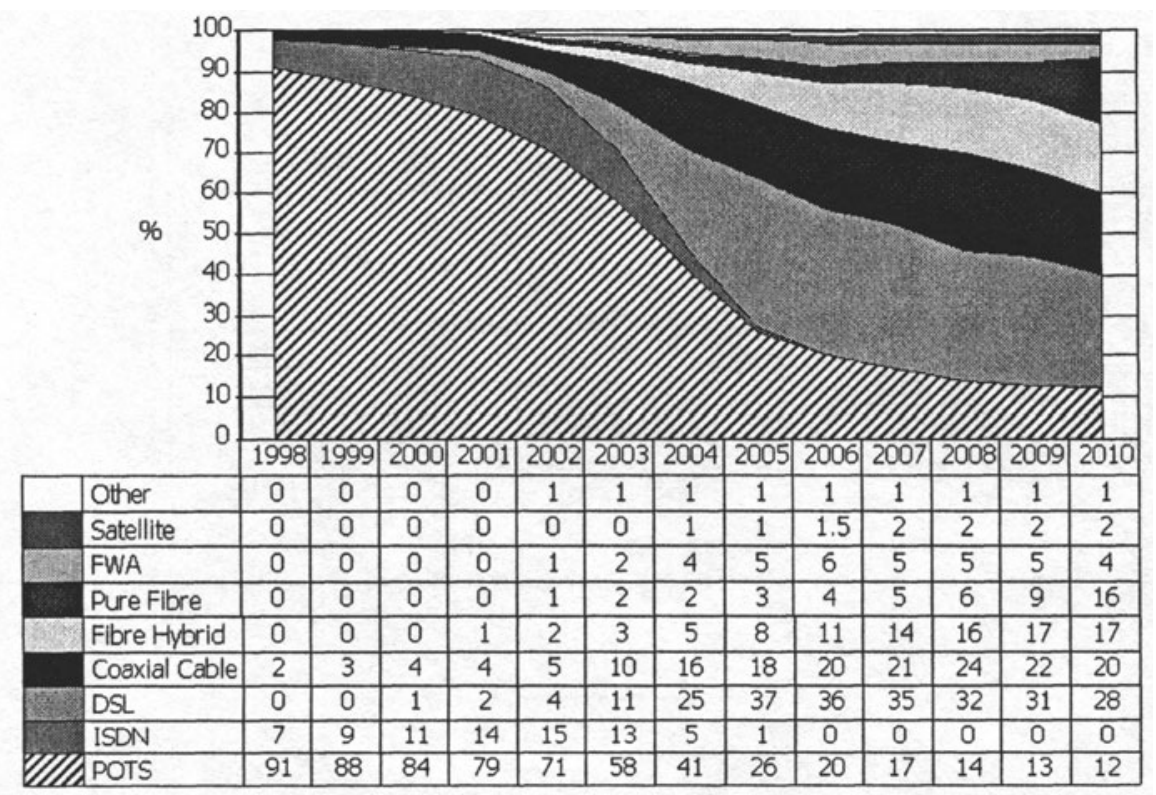

Figure 12. Predicted market share of access platforms to EU homes / small medium enterprises (SME)

The predicted growth of household broadband, DSL and broadband cable in the EU 15, US and Japan is plotted in Figure 13. Remarkable is the fact that Europe and Japan will not be able to make up the technological gap regarding broadband access in the near future. $22 \%$ of US households will possess broadband access, whereas just $11 \%$ of European homes will own a broadband access in 2003.

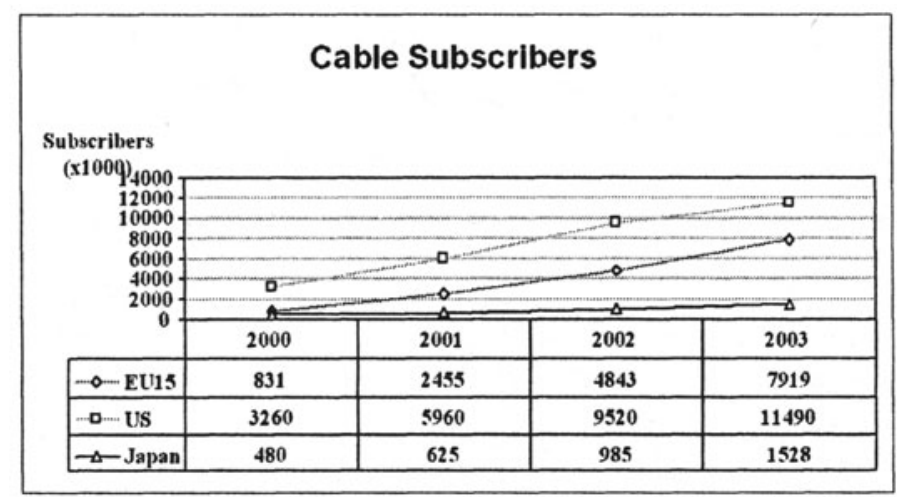



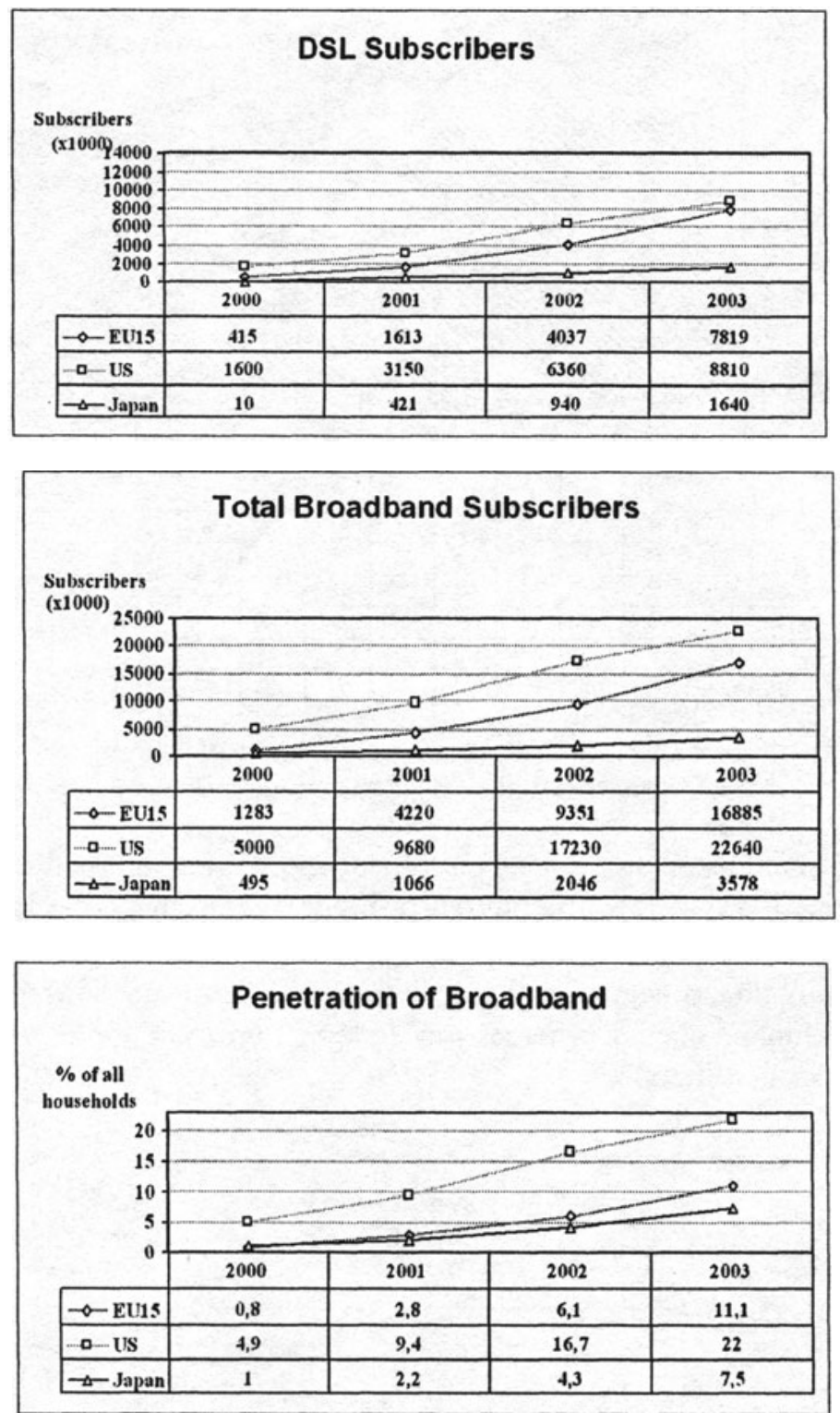

Figure 13. Predicted growth of household / SME

Broadband, DSL and Broadband Cable in the EU 15, US and Japan

\section{THE E-BUSINESS IMPACT ON ICT}

E-business is more than the latest rage in information technology, it is the new order. For the purpose of building a picture of the impact of e-business 
on the ICT market and consequently on the global economy at macro- and micro-economic level, we first go through the market trends of e-business.

\subsection{E-business Consolidation}

After reaching its euphoric peak in mid 2000, e-business suffered a drastic downfall by the end of 2001. Moreover, the e-business industry will now enter a phase of massive problems and disillusionment. According to latest studies even $75 \%$ of all projects in the field of e-business may fall short of their expectations in the near future. This downfall will hit 'brick and mortar' as well as dotcom companies. However, the temporary crash of ebusiness does not implicate an absolute decline of e-commerce, eprocurement, or e-business in general. In fact, the exact opposite will occur. The reasons for the collapse of e-business projects in the next years are mainly immature business models, strategic errors, and implementation problems. The aftermath of this development will lead to 'real' e-business companies approximately in 2004. Between 2006 and 2008 the majority of enterprises will have completed their economic metamorphose to so-called 'brick and click' companies.

Figure 14 provides a snapshot of the inevitable cycle of hype and disillusionment that accompanies the technological path to e-business maturity. It consists of the following phases:

Technology trigger: A breakthrough, public demonstration, product launch, or other event generates significant press and industry interest. Quantum computing and other non-silicon computing techniques are still firmly in the research laboratories, but will represent the future of computing once today's chip manufacturing techniques hit the limits of physics. Audio mining will make previously untapped data sources (e.g., recorded meetings, phone calls, and conversations) available for search and analysis through speech-to-text technology. Synthetic characters will enable users to interact in natural language with a computer-generated personality to access online information and services, although today's characters are severely limited in scope. Digital ink, which allows dots of ink to be flipped like screen pixels, will convert a broad range of surfaces (e.g., packaging and signs) into rewriteable displays. Biometric identification, which uses physical characteristics (e.g., face and voice) for authentication, is being boosted by Microsoft's adoption of the BAPI standard. Voice portals, which provide automated speech access over the phone to information and messaging 
services, will explode onto the market during the next year, with hundreds of new, typically free, branded offerings.

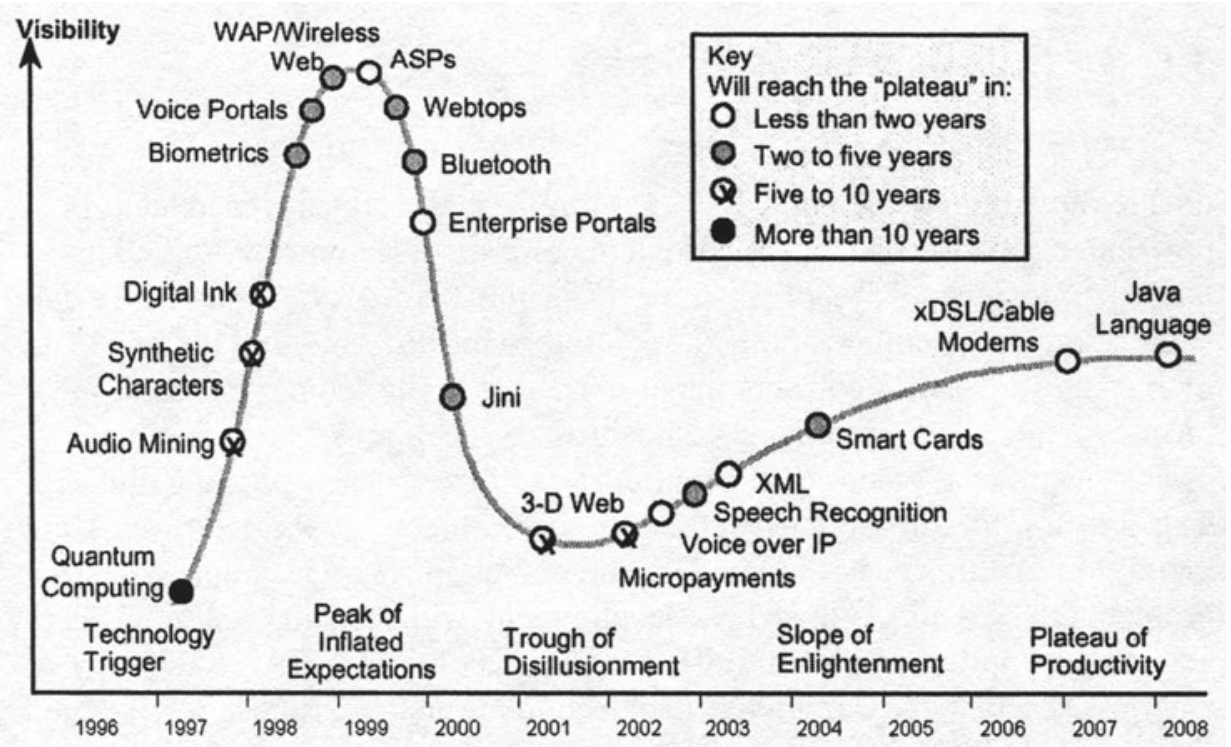

Figure 14. E-business hype-cycle

Peak of inflated expectations: During this phase of over-enthusiasm and unrealistic projections, a flurry of well-publicized activity by technology leaders results in some successes but more failures as the technology is pushed to its limits. The only enterprises making money are conference organizers and magazine publishers. Wireless Web/WAP phones are available from leading vendors as a means of accessing Web information from mobile devices. ASPs, which outsource the development and hosting of application software and hardware, are springing up in every area of IT, from SAP to speech recognition. Webtops are virtual desktops resident on the network that give users access to their information and applications from wherever they gain access to the Internet.

Trough of disillusionment: Because the technology does not live up to its over-inflated expectations, it rapidly becomes unfashionable and the press abandons the topic. Bluetooth chips are beginning the difficult path toward mass adoption. Enterprise portals have combined personalization, search and document management capabilities, but offer fundamentally nothing new. Jini, which promises true plug-and-play for computing peripherals, still needs to be translated into meaningful products. 3-D Web technologies for visualizing and exploring objects and spaces are still hampered by bandwidth and a shortage of compelling applications. 
Slope of enlightenment: Focused experimentation and solid hard work by an increasingly diverse range of organizations lead to a true understanding of the technology's applicability, risks and benefits. Commercial off-the-shelf methodologies and tools become available to ease the development process. Climbing the Slope. Micropayments are overcoming the disillusionment surrounding e-wallet schemes and reemerging in new forms, such as billing to existing payment aggregators (e.g., phone companies and ISPs). Voice over IP is reaching levels of quality needed for applications such as integrated phone/Web contact centers. Speech recognition is providing significant paybacks in automating telephone transactions (but is not widely accepted on the corporate desktop). $\mathrm{XML}$ is broadly accepted as a standard in Web products for data and content exchange. Smart card technology is well-established in some countries for mobile phone, prepayment and bank card applications, although trailing in the United States. Cable modems and xDSL are well established as viable options for high-bandwidth connectivity.

Plateau of productivity: The real-world benefits of the technology are demonstrated and accepted. Tools and methodologies are increasingly stable as they enter their second and third generation. The final height of the plateau varies according to whether the technology is broadly applicable or benefits only a niche market.

Success on the long run can only be assured by a strictly integrative ebusiness concept. Thus, enterprises should be selectively aggressive in the early adoption of technologies with the potential for high impact, but should ride out the hype-cycle for less-critical technologies.

\subsection{Top E-Business Trends}

E-business market research predicts a rather balanced growth for the next two years, whereas an optimal growth rate is expected thereafter. Figure 15 presents the market development of e-business until the year 2004. The driving force of the e-business evolution is the Business-to-Business (B2B) sector, which is expected to exceed the 4.000 Billion Euro limit by 2004 . 


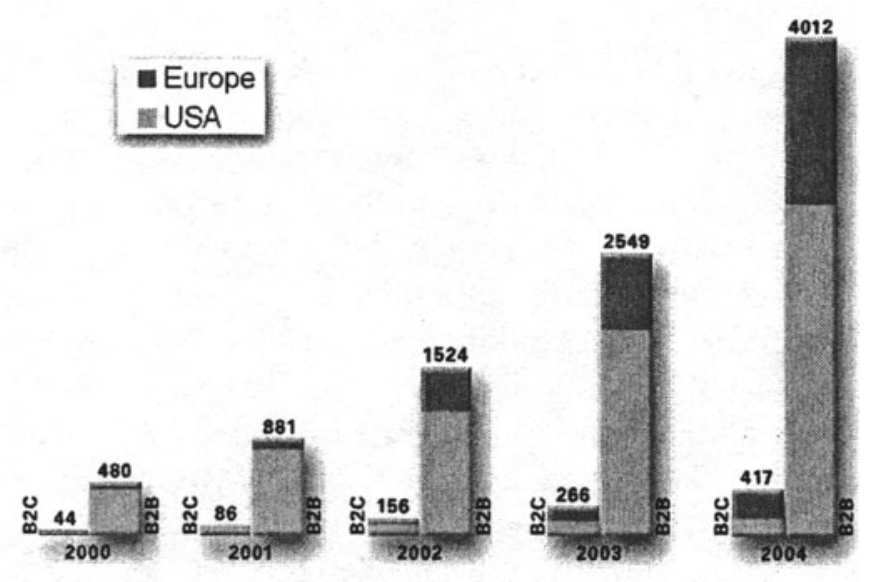

Figure 15. B2B dominates the e-business evolution

The following part presents a summary of the most important trends determining the e-business market in the near future. Major companies are preparing to greatly expand their investment in e-business. Thus, the next three years will see rapid growth of companies using e-business to improve the quality of customer and supplier interactions. The benefits of doing business electronically will attract increasing interest as companies realize the importance of not merely doing business better (e-commerce) but doing business differently (e-business). Priorities will focus on information sharing to better serve customers. Companies will strive to optimize customer relationships, creating the loyalty that is the basis for efficient profit growth and competitive advantage. E-business security and privacy are critical to companies' success in attracting online business. Especially security is still an open issue. It will either be a big business chance for R\&D companies or it will herald the end of e- / m-business. Companies will be expected to explain their policies for protecting customers from risk and disclosure of personal information. The realization that a successful e-business is more about business and less about technology will grow as more executives grapple with the pressure to join electronic with traditional business structures. Priorities will include the tax, legal, risk and compliance issues that will allow companies to outdistance rivals. CEOs may find themselves managing the regrouping of company functions in response to increasingly flexible information and distribution channels and the disassembly of the value chain. PWC studies confirm that executives are savvy to the sweeping implications of e-business and are becoming educated themselves about their options. Companies want to see more successful e-business examples before embarking on such endeavors themselves. These e-business role models are likely to come from information-based industries such as high tech and financial services. E-business will continue to be a "net cash out" proposition 
as companies build the infrastructure needed to succeed. The future winners will continue to invest while finding ways to evolve their existing investments in channels and infrastructure to become more e-business dominant. Effective partnerships and alliances are a key feature of internetbased organizations. They can lower start-up costs and share risks while accelerating progress toward becoming a true e-business. World Wide Web achieves global status. This is largely linked to the increasing globalization of business, which relies on an Internet environment where there are no geographic boundaries

\section{M-BUSINESS}

Mobile business is simply Internet + Wireless + E-business. This means changing the paradigm from a tethered PC-centric model towards a mobileperson-centric model. That is to say availability anytime and anywhere.

\subsection{Mobile Device Market}

Mobile business is going to raise the need for small, but increasingly powerful mobile devices. Figure 16 gives an overview of the market segmentation in the field of digital terminal mobile devices.

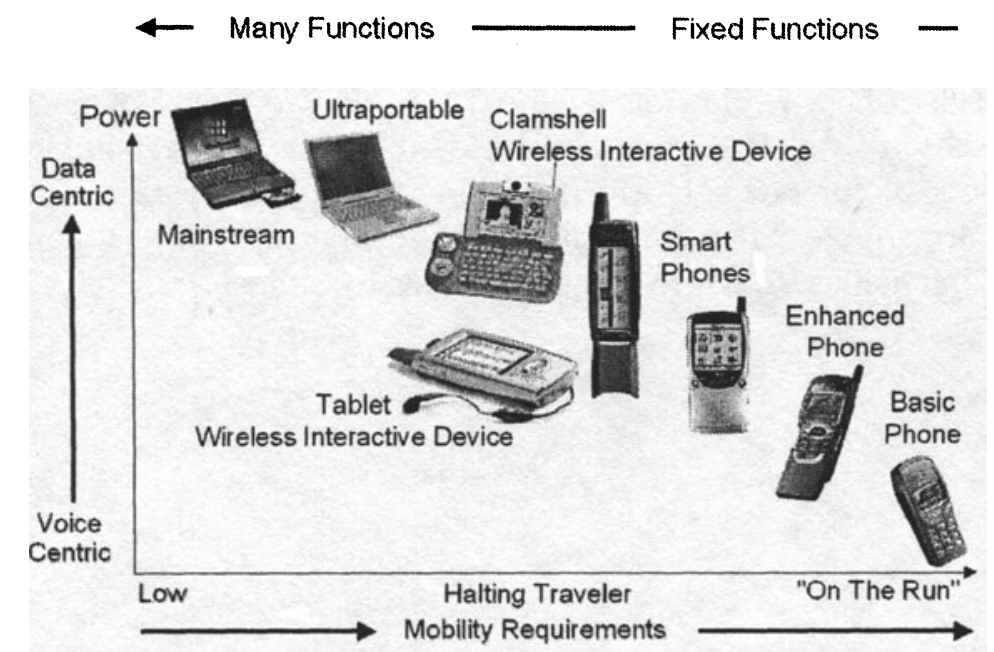

Figure 16. Mobile device market segmentation 


\subsection{Mobile Subscriber Market}

The number of mobile subscribers worldwide is increasing very rapidly. It is expected that by the year 2004 the number of mobile subscribers will exceed the number of fixed mainlines worldwide. Just one year later the number of mobile internet users will outrun the count of fixed internet users. Finland was the first country in the world (1998), where the mobile subscriber density exceeded the fixed subscriber density. Figure 17 displays the mobile subscriber density and the fixed subscriber density for selected countries.

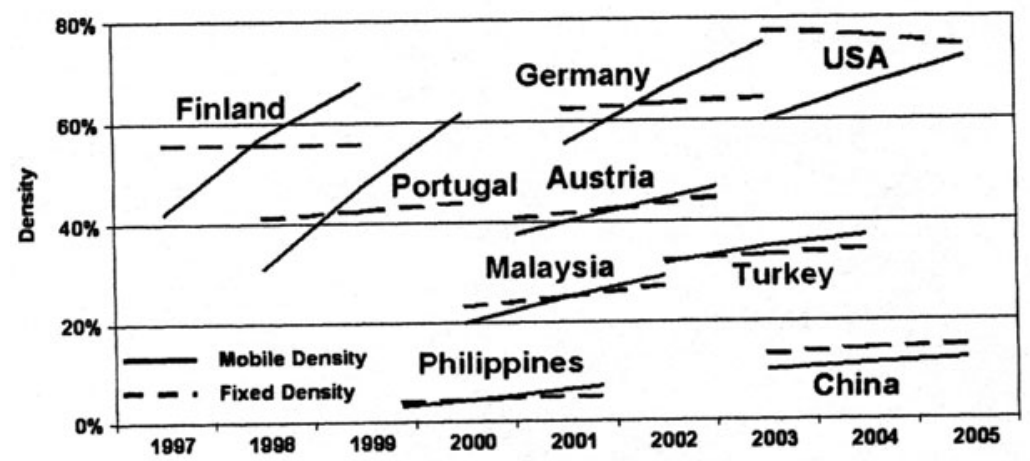

Figure 17. Selected mobile versus fixed densities

Despite the global economic slowdown the market for mobile subscribers will continue to grow and will reach a high of approximately 1.4 Billion mobile subscribers worldwide. Figure 18 displays the development of the mobile subscriber market from the year 2000 to 2005. Interesting fact is that the number of contract subscribers will be outrun by prepaid customers by 2005. Furthermore Figure 18 shows the overgrowth of the number of wireless internet users, which is mainly driven by m-business. 


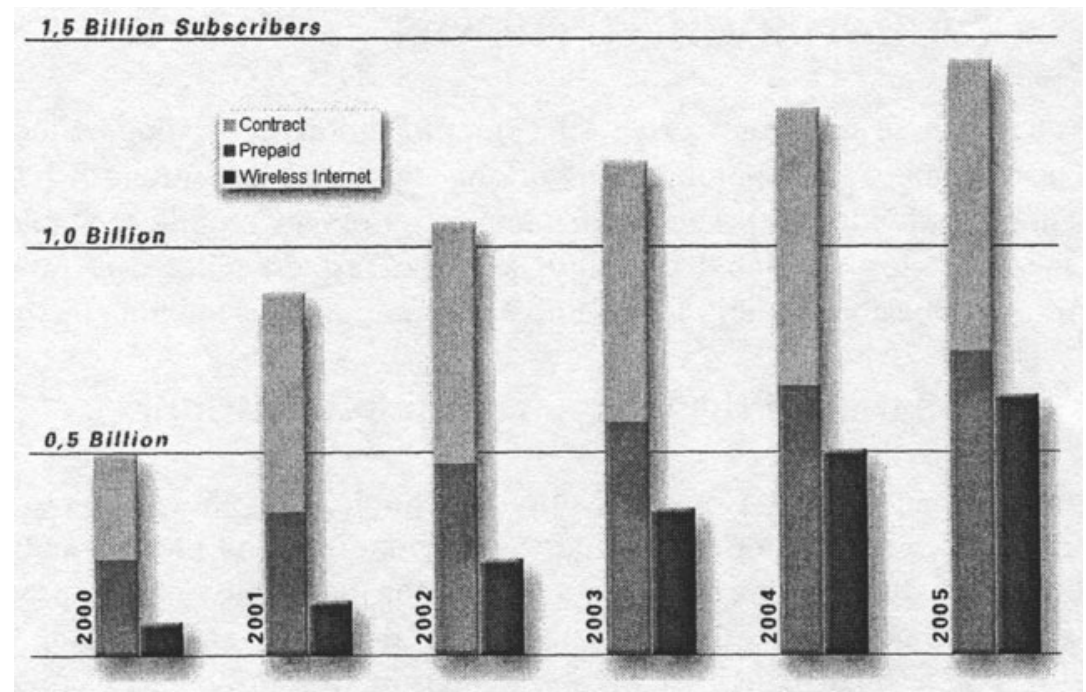

Figure 18. Evolution of mobile contract and mobile prepaid subscribers versus wireless internet users

\subsection{Mobile Phone Market}

These are challenging times for the mobile phone industry. After a decade of surging growth, global sales of handsets fell for the first time in the second quarter of 2001 by more than 8 per cent, as operators reduced handset subsidies and consumers held back from making replacement purchases. But if the current state of the market is a concern for manufacturers, the future looks terrifying - or at least terrifyingly difficult to predict. Manufacturers are having to grapple with the technological issues involved in designing the new handsets for GPRS and 3G systems without knowing how much attention each should receive relatively, with little hard data on how many units the operators will need or when they will want them, and a growing sense that end-users are quite happy with the functionality of their existing phones. On top of all that, those handset operators that are also in the infrastructure business know that $3 \mathrm{G}$ may arrive sooner than later only if financially-pressed network operators share equipment, thus reducing equipment sales. Steering a course through all this will be a severe test of manufacturers' product development, design and production strategies. The first hurdle is expected with Christmas 2001. Shipments of GPRS have already been delayed, and are urgently needed by operators and retailers if the holiday season is to provide the hoped-for boost to handset sales. 


\section{TECHNOLOGICAL TRENDS}

Essential three directions drive telecommunications technologies today: (1) The advances in photonic networking with an evolution of everincreasing bandwidth; (2) The tremendous progress in mobile technology with the evolution to global mobility; (3) The fast evolving pace of IPnetworking as basic paradigm for an universal, ubiquitous switching method.

\subsection{Evolution of the Ever-increasing Bandwidth}

The Information and Communication Technology is the most rapidly growing market in the world. The increase of mobility and the demand for application bandwidth were the driving forces for the evolution of improved bandwidth technologies. Starting with some $100 \mathrm{kbit} / \mathrm{s}$ and reaching the range of Tbit/s. The main part of this evolution was provided by optical networks which now cover the globe. The amount of data that can be carried in each strand of fibre in these networks is growing. Dense Wavelength Division Multiplexing allows 40 channels of $40 \mathrm{Gbit} / \mathrm{s}$ to be sent down on one fibre - enough to transmit the whole of the movie 'Gladiator' in a fraction of a second. Every year, more wavelengths (or colors) will be available and each will carry more information. There is enough raw transmission capacity, at least on main trunk routes, to satisfy the world's traffic requirements several times over. For future data applications the available peak bit rate will be more important than the pure average bit rate. A user who wants to download a file will do that not permanently but when he tries to do it, he will not be willing to wait too long. As a forecast the average bit rate per application will be 0,1 to $0,5 \mathrm{Mbit} / \mathrm{s}$ in 2005 with streaming applications excluded (they will need a much higher bit rate). The corresponding peak bit rate per application should be 2 to $5 \mathrm{Mbit} / \mathrm{s}$. Bit rate demands and the capability of the technology are interrelated. Whenever high bit rates can be provided at low cost, a corresponding application will appear and cause, after some time needed to get used to it, a demand for an even higher rate. This raises the question for the limit of that process. A preliminary answer is that for a single application all information which can be received by an person can be coded into 100 to $200 \mathrm{Mbit} / \mathrm{s}$. Estimations however are highly uncertain and cannot be used as the single criteria to decide about future technology. This evolution will never satisfy the everlasting bandwidth demands. Figure 19 presents a survey of the milestones development in the core, fixed, mobile and satellite networks. 


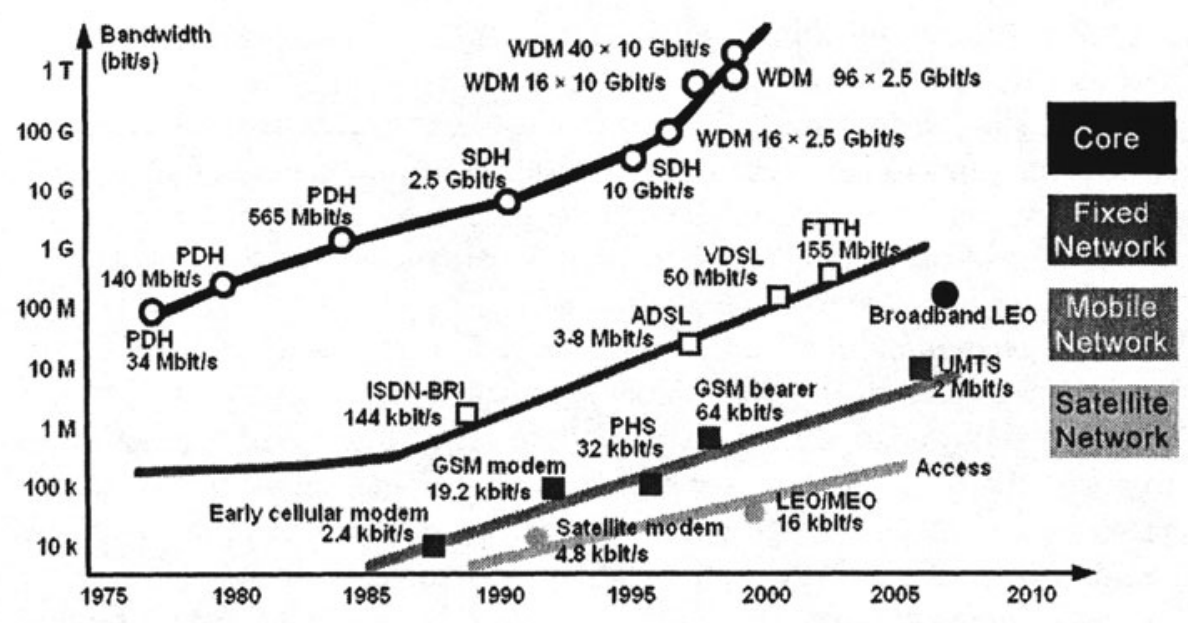

Figure 19. Evolution of the ever-increasing bandwidth

\subsection{Evolution of Mobility}

The telecommunications industry has developed in the last decade a number of new solutions to handle the complexity introduced within the network by the growing demand for new services. For mobile communication networks, new radio technologies in UMTS (Universal Mobile Telecommunication System) make roaming more powerful but at the same time more complex. New core network technologies, such as packet switching and IP multimedia, complicates roaming also from a service control perspective. Continuing the success of roaming achieved in GSM is a significant challenge and will be dependent on continued efforts by operators and manufacturers in creating the necessary high-quality standards for the future mobile communication system. The main emphasis is on mobile networks but there are still many benefits that accumulate both to the customer and to a combined fixed/mobile network operator, from attempting to share common infrastructure components between networks. Seamless services have been much discussed but the concept runs into problems due to detailed network disparity, different network management, and product portfolios. While not necessarily attempting to use the same network components between fixed and mobile networks, it is clear that there are many similarities in the technologies adopted by the fixed network. In the context of mobile telecommunication, three important aspects characterize a concept of personal communication support (PCS): support of mobility, including both terminal and personal mobility, service customization, and service integration/interworking. PCS is an improved form of universal 
personal telecommunication (UPT) and will allow a UMTS user to customize his call handling policies.

Personal mobility is the ability of the end user to access services and to receive invitations to services on different terminals in different locations. In contrast, terminal mobility is the ability of a moving terminal to access telecommunication services from different locations and the capability of the network to identify and locate that terminal.

Service customization enables a user to define various conditions for his subscribed services by many parameters, such as time-dependent, servicedependent, and user-dependent call handling. The customized parameters of a user are usually stored in a user profile, which defines all services to which the user has access and usage, the way in which service can be handled, and all other user-configurable communication properties.

Service integration/interworking is required because UMTS will support personal mobility where the user can register from many kinds of terminals with various capabilities. Therefore, it will be necessary for the PCS to be able to convert an incoming call to adapt with the capabilities of the terminal at which the user is currently registered. Basically, the user can configure a set of attributes that define how an incoming call is to be handled by the system. This functionality is similar as in the virtual home environment (VHE).

Basically, the VHE concept in the third-generation mobile communication network like UMTS will allow the support service mobility, also called service roaming and service portability, respectively. The basic idea is that a roaming user will be able to customize his own environment individually at different terminals within different networks. The VHE will permit service mobility for the user, which means that the user takes his subscribed and customized services with him while he is roaming. VHE and its associated tools provide an ideal platform for use by independent entities (such as private networks and Intranets) that seek to deliver telecommunications-based services to their subscribers. The technology also enables to create totally new content and value added services. The flexibility introduced by VHE not only increases the range of services that can be offered to subscribers and users, but also allows them to be tailored to different user types and market sectors, even down to an individual user. The personal profiles can be extended beyond "any one, any time, any place" so that the services are consistent but also able to adapt to the users' circumstances as well as the capabilities of their terminals and access networks. Some of the requirements to realize in such a VHE environment are: (1) The capabilities of any available terminal within any accessed network to be sufficient for performing the services of the registered user; (2) Service conversion that can be useful to provide a service on a terminal, 
which cannot support the original service. The VHE will enable a visited network to obtain information about the user's service profile as well as other information, such as the user's personalized service profile and identification of the service capabilities needed for the execution of service profile-specific services. The VHE can be seen as a (middleware) layer, which hides the concrete network capabilities and differences from the user and provider systems capabilities.

The third generation (3G) mobile networks are under development to support for diverse wireless access technologies, innovative applications that can seamlessly integrate data and voice, and service portability and mobility. These new activities in IETF (Internet Engineering Task Force) and the 3G communities indicate that future mobile telecommunication networks are rapidly migrating toward an all-IP technology. The all-IP-based 3G mobile network architectures can be viewed as consisting of a core backbone network (IP network) connected to many overlay networks, each with its own specific characteristics and different access technologies. The universal wireless communication networks (UWCN) consist of overlaid super-cells (via satellite), macro-cells, micro-cells and pico-cells that cover widely varying geographic areas, ensure global personal connectivity, and support different data rates and access technologies for mobile terminals within them. A key role of IP in 3G mobile networks will probably be the provision of efficient and cost-effective interworking between overlay networks. On the other hand, the availability of advanced IP mobility protocols makes Internet technology attractive for both interworking between different networks and for the definition of new mobile network architectures.

\subsection{Evolution of IP-Networking}

The largest network providing broadband capabilities is the Internet, and it is believed that future broadband networks will be based mostly on IP technology. The set of requirements for a generic broadband network is larger than the set of capabilities provided in today's Internet. Thus, it became necessary to eliminate some weaknesses by enhancing the internet protocols. An important new feature is the provisioning of quality of service (QoS), which is required for real-time services such as voice and video conferencing. Two protocols have been developed for QoS provisioning in the Internet: integrated services and differentiated services. The integrated services protocol is primarily designed for the access domains, whereas the differentiated services protocol is better suited for the core domain. QoS 
routing and bandwidth management capabilities are provided by the Multiprotocol Label Switching (MPLS) protocol.

\section{CONCLUSIONS}

The analytical market study of the development of the information and communication technology (ICT) showed the different trends and their impact on the information communication networks and consequently smart networks. The paper showed that, despite the current global economic difficulties in almost every single industry, there are still a lot of business opportunities within the ICT industry and there are enormous business chances to adapt, to optimize, and to exploit in the already existing networkinfrastructures in Eastern Europe as well as in the Third World. Techologically, three directions drive telecommunications today: photonic networking, global mobility, and IP-networking.

\section{REFERENCES}

[1] Harmen R. van As, Telecommunication Network Intelligence. Kluwer Academic Publishing, 2000.

[2] Ravi Kalakota and Marcia Robinson, M-Business: The Race to Mobility. McGraw-Hill, 2002.

[3] European Information Technology Observatory, 2001.

[4] Next Generation Optical Networks, The Convergence of IP Intelligence and Optical Technologies. Prentice Hall PTR, 1st edition, August 2001.

[5] Jerry Wind, Vijay Mahajan and Robert Gunther, Convergence Marketing: Strategies for Reaching the New Hybrid Consumer. Prentice Hall, 1st edition, October 2001.

[6] Stewart McKie, E-Business Best Practices: Leveraging Technology for Business Advantage. John Wiley \& Sons, April 2001.

[7] Hacking Exposed: Network Security Secrets \& Solutions. McGraw-Hill, 3rd Edition, September 2001.

[8] H. Aghvami, B. Jafarian, A Vision of UMTS/IMT-2000 Evolution, IEE Elec. \& Comm. Eng. Journal, Vol. 12, No. 3, June 2000, pp. 148-152.

[9] M. D. Cookson and D. G. Smith, 3 G service Control, BT Technol. J, Vol. 19, No 1, Jan. 2001, pp. 67-79. 
[10]Bruce S Davie, Yakov Rekhter, MPLS. Technology and Applications, Morgan Kaufmann, 2000.

[11]T. Li: MPLS and the Evolving Internet Architecture, IEEE Communications Magazine, Vol. 37, No. 12, Dec. 1999, pp. 38-41.

[12] 3GPP home page: http://www.3GPP.org.

[13]Datamonitor: http://www.datamonitor.com/

[14]European Information Technology Observatory: http://www.eito.com/

[15] Financial Times: http://news.ft.com/

[16] Forester Communications: http://www.forester.net/

[17] Gartner Group http://www4.gartner.com/

[18] Global Internet Project: http://www.gip.org/

[19] IEEE: http://www.ieee.org/

[20] IEEE Communications Society: http://www.comsoc.org/

[21] International Data Corporation: http://www.idc.com/

[22] International Engineering Society: http://www.iec.org/

[23] International Softswitch Consortium: http://www.softswitch.org/

[24] Internet Society: http://www.isoc.org/

[25] Internet Surveys: http://www.nua.com/

[26] InterPlanetary Network Special Interest Group: http://ipnsig.org/

[27] Mobile Wireless Internet Forum (MWIF), http://www.mwif.org/

[28] Next Generation Internet: http://www.ngi.gov/

[29] OVUM: http://www.ovum.com/

[30] Smau Italy: http://www.smau.it/

[31] Telco Research: http://www.peregrine.com/

[32]Tele Dot Com: http://www.teledotcom.com/

[33] Total Telecom: http://www.totaltele.com/

[34] WorldCom: http://www.worldcom.com/ 